\title{
Significant diaphragm elevation suggestive of phrenic nerve injury after thoracoscopic lobectomy for lung cancer: an underestimated problem
}

\author{
Luigi Ventura ${ }^{1,2 \#}$, Weigang Zhao ${ }^{1,3 \#}$, Tangbing Chen ${ }^{1}$, Zhexin Wang ${ }^{1}$, Jian Feng ${ }^{1}$, Zhitao Gu ${ }^{1}$, Chunyu Ji ${ }^{1}$, \\ Wentao Fang ${ }^{1}$
}

${ }^{1}$ Department of Thoracic Surgery, Shanghai Chest Hospital, Jiaotong University Medical School, Shanghai, China; ${ }^{2}$ Thoracic Surgery, Department of Medicine and Surgery, University Hospital of Parma, Parma, Italy; ${ }^{3}$ Department of Thoracic Surgery, the Sixth People's Hospital, Jiaotong University Medical School, Shanghai, China

Contributions: (I) Conception and design: W Fang, L Ventura; (II) Administrative support: T Chen, Z Wang; (III) Provision of study materials or patients: W Fang, C Ji, Z Gu; (IV) Collection and assembly of data: W Zhao, J Feng; (V) Data analysis and interpretation: W Fang, L Ventura; (VI) Manuscript writing: All authors; (VII) Final approval of manuscript: All authors.

\#These authors contributed equally to this work.

Correspondence to: Wentao Fang. Department of Thoracic Surgery, Shanghai Chest Hospital, Jiaotong University Medical School, 241 Huaihai Road West, Shanghai 200030, China. Email: vwtfang@hotmail.com.

Background: Phrenic nerve injury (PNI) during lung cancer surgery, without apparent nerve section or damage, is still not well-studied. The aim of our study is to find an easy and objective way to evaluate a significant diaphragm elevation (SDE) suggestive of inadvertent PNI and its incidence and impact on lung cancer patients undergone video-assisted thoracoscopic surgery (VATS) lobectomy.

Methods: Extent of diaphragm elevation was first examined on chest X-ray in a cohort of patients with invasive thymoma in whom phrenic nerve was intentionally transected. The result was then used as the criterion to diagnose a SDE suggestive of PNI in another cohort of VATS lobectomy patients. Fluoroscopy test was used to validate the results. Spirometry test was repeated to evaluate pulmonary function loss after surgery.

Results: Diaphragm elevation was $24.24 \% \pm 6.2 \%$ in 22 invasive thymoma-patients, with $30 \%$ elevation adopted as the criterion to diagnose SDE suggestive of PNI. In 753 VATS lobectomy patients, 56 (7.4\%) were diagnosed of SDE. On Fluoroscopy test, diaphragm movement was significantly less in patients with diaphragm elevation $>30 \%$ than those without $(5.0$ vs. $11.0 \mathrm{~mm}, \mathrm{P}=0.003)$, together with a significantly smaller diaphragm movement ratio on the operation (OP) side than on the contralateral side (17\% vs. $42 \%$, $\mathrm{P}=0.018$ ). Although no difference in postoperative complications was found, reduction in FEV1, FVC, and DLCO was significantly greater in patients with a SDE than those without $(\mathrm{P}=0.009)$.

Conclusions: Patients with more than 30\% diaphragm elevation after VATS lobectomy is highly likely to have PNI and should undergo fluoroscopic validation. Inadvertent PNI during VATS lobectomy is an underestimated phenomenon and is associated with significantly greater loss of pulmonary function.

Keywords: Phrenic nerve injury (PNI); video-assisted thoracoscopic surgery (VATS); lobectomy; lung cancer; thymectomy

Submitted Apr 07, 2020. Accepted for publication Aug 03, 2020.

doi: $10.21037 /$ tlcr-20-540

View this article at: http://dx.doi.org/10.21037/tlcr-20-540 


\section{Introduction}

Phrenic nerve injury (PNI) is a well-noted complication after cardiac (1-4) and sometimes also after thymic surgery $(5,6)$. On the contrary, scarcely has attention been paid to inadvertent PNI during lung cancer surgery, with few cases reported (6). Although some diagnostic tools have been proposed for detecting PNI (7-13), they are not readily available in most of the cardio-thoracic surgery units. During surgical resection for lung cancer, unexpected significant diaphragm elevation (SDE) on post-operative chest film is often the first sign suggesting PNI. But this is mainly subjective and without a welldefined criterion for quantitative measurement (2-5). Upon fluoroscopic examination, a paralyzed diaphragm with absent or paradoxical movement is considered diagnostic of the presence of PNI. Video-assisted thoracoscopic surgery (VATS) is now the recommended approach for surgical management of early-stage lung cancers, with well demonstrated advantages over open thoracotomy $(14,15)$. With different techniques and instruments used for these two approaches, new problems not commonly seen in open surgery may emerge, including PNI. The aim of this study is therefore to quantitatively define an SDE suggestive of PNI and then to evaluate its incidence and functional impact on lung cancer patients undergone VATS lobectomy. We present the following article in accordance with the STROBE Guideline (available at http://dx.doi. org/10.21037/tlcr-20-540).

\section{Methods}

This is a retrospective cohort study using a prospectively kept database. All cases included in this study were operated on by a single team of surgeons (WF). Extent of diaphragm elevation was first evaluated in a cohort of patients with stage III thymic tumors in whom phrenic nerve was intentionally co-resected because of tumor invasion. The results were then used to evaluate diaphragm elevation and potential PNI in another cohort of patients undergoing VATS lobectomy for lung cancers.

All procedures performed in this study were in accordance with the Declaration of Helsinki (as revised in 2013). The study design was approved by the Local Ethics Committees. All patients gave a written informed consent for the surgical treatment and were aware that all information could be used anonymously for scientific purpose only.

\section{Phase I: quantitative evaluation of SDE suggestive of PNI}

Extent of diaphragm elevation was first measured on chest $\mathrm{X}$-ray before and 6 months after surgery in thymic tumor patients cohort. Both chest films were taken in posteroanterior projection, on full inspiration and with the patient in standing position. The distance between the highest point of the diaphragm and the apex of the chest before (DB $=$ distance before $)$ and after surgery $(\mathrm{DA}=$ distance after $)$ was measured and recorded. Extent of diaphragm elevation was calculated by the following formula: [(DB-DA)/ $\mathrm{DB}] \times 100 \%$. Fluoroscopy test was performed to validate the diaphragm movement on both the operation (OP) and the non-operation (NOP) sides and to calculate diaphragm movement ratio, defined as diaphragm movement on the OP side divided by that on the NOP side. Considering that there would be loss of lung volume after lobectomy, the above result in mean value + standard deviation (SD) was then taken as the criterion indicating an SDE potentially related to PNI in lung cancer patients undergone VATS lobectomy.

\section{Phase II: evaluation of SDE suggestive of PNI after VATS lobectomy}

From a prospectively kept database, patients having VATS lobectomy for lung cancers from January 2012 to December 2016 were retrieved for this study. Only patients with no previous thoracic surgery and no pre-treatment for lung cancer were included. Patients in whom phrenic nerve was transected during surgery because of tumor or lymph node invasion and those converted from VATS to open surgery were also excluded. Since only de-identified information was used for the study, written consent was waived by the hospital IRB. VATS lobectomy was fulfilled through a standard three-hole approach. Hilum mobilization was carried out using energy devices including hook electric cautery or harmonic scalpel. Lymph nodes in hilum were always dissected together with the lobe resected. In patients with lesions appearing as ground-glass opacities, mediastinal nodes were systemically sampled. Skeletonised mediastinal nodal dissection was performed only in patients with solid or sub-solid lesions. Extent of diaphragm elevation in every patient was measured according to the methods described in the first phase of the study. To rule out potential influences of pleural effusion, atelectasis, or pneumonia in the early post-operative period (1), comparison of diaphragm levels was carried out using chest $\mathrm{X}$-rays before and 6 months after 

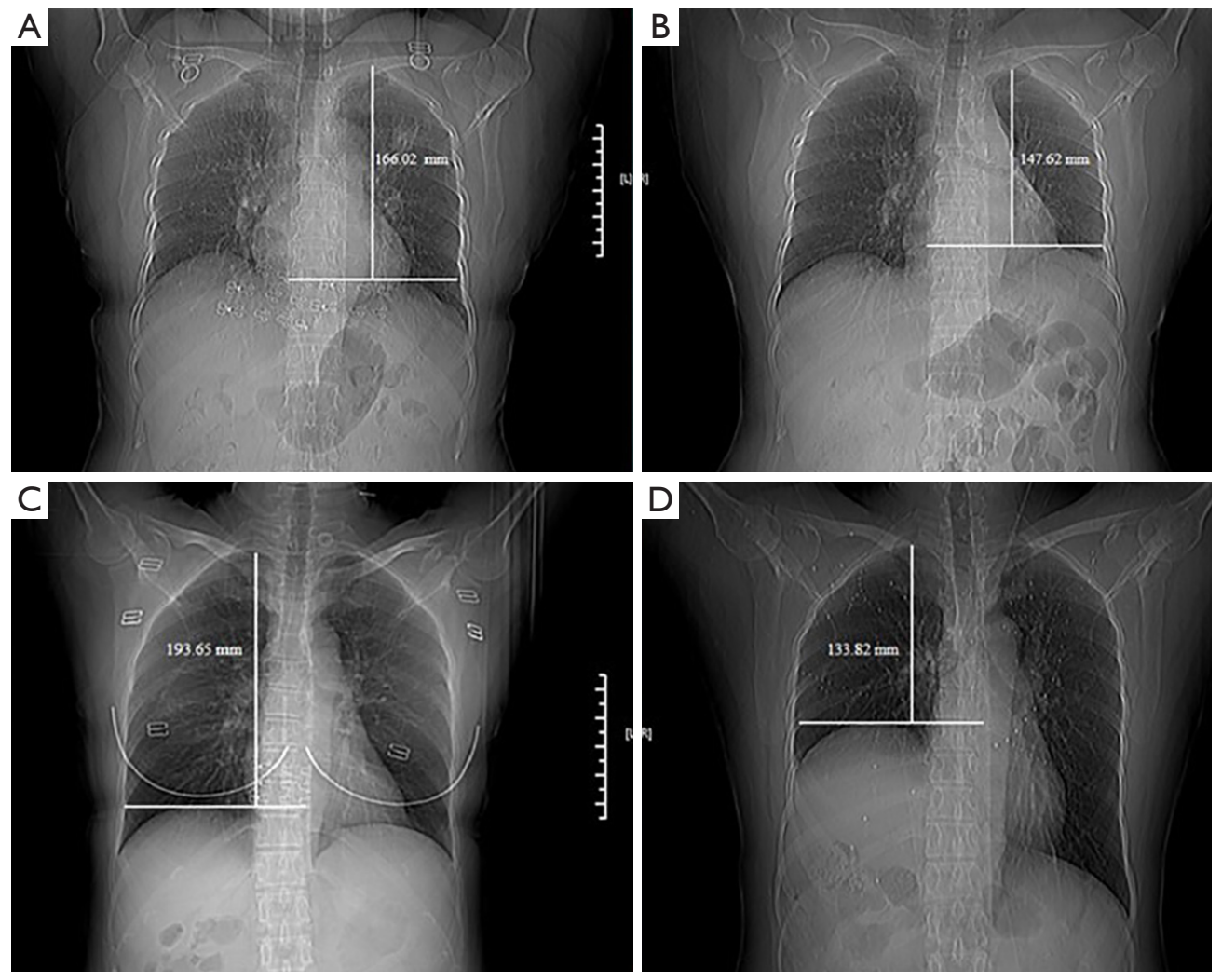

Figure 1 Typical chest film presentations of patients with and without significant diaphragm elevation after VATS lobectomy for lung cancers. (A,B) Chest films of a 54-year-old female patient before and after left upper lobectomy. The distance between the apex of the diaphragm and the apex of the chest on pre- and post-operative chest X-ray was $166.02 \mathrm{~mm}$ (A) and $147.62 \mathrm{~mm}$ (B), respectively. Diaphragm elevation calculated by [(DB-DA)/DB] $\times 100$ was $11.1 \%$. (C,D) Chest films of a 51-year-old female patient before and after right upper lobectomy. The distance between the apex of the diaphragm and the apex of the chest, on pre- and post-operative chest X-ray was 193.65 $\mathrm{mm}(\mathrm{C})$ and $133.82 \mathrm{~mm}(\mathrm{D})$, respectively. Diaphragm elevation calculated by [(DB-DA)/DB]×100 was $31 \%$.

surgery (Figure $1 A, B, C, D$ ). SDE was diagnosed according to the criterion established through Phase I of the study. Fluoroscopy test was again used to verify SDE diagnosed by chest $\mathrm{X}$-ray and to calculate diaphragm movement ratio as mentioned above. Spirometry test was carried out as a part of preoperative workup in all patients, and also at 6 months after surgery in those patients who did not receive any adjuvant therapy when they came to the outpatient clinic for follow-up (16). The results were expressed as absolute and predicted values preoperatively and as the rate of decline postoperatively. Surgical outcomes and spirometry changes were then compared between patients with or without SDE after surgery.

\section{Statistical analysis}

Quantitative data were expressed as mean $\pm \mathrm{SD}$. Continuous variables were compared using Student's $t$-test or MannWhitney $\mathrm{U}$ test, and categorical variables were analyzed by $\chi^{2}$ test. A $\mathrm{P}$ value $<0.05$ was considered statistically significant. All statistical analyses were calculated using the SPSS software version 19.0 (SPSS Inc., Chicago, IL, USA).

\section{Results}

\section{Diagnostic criterion for SDE suggestive of PNI}

Extent of diaphragm elevation was found to be $24.24 \% \pm 6.2 \%$ in 22 invasive thymic tumor patients with phrenic nerve intentionally transacted during thymectomy. Big wedge resection of the lung was also carried out in all of the patients because of tumor invasion. The upper limit (mean value $+\mathrm{SD}=30 \%$ ) was adopted as the criterion for characterizing an SDE potentially due to PNI after VATS 

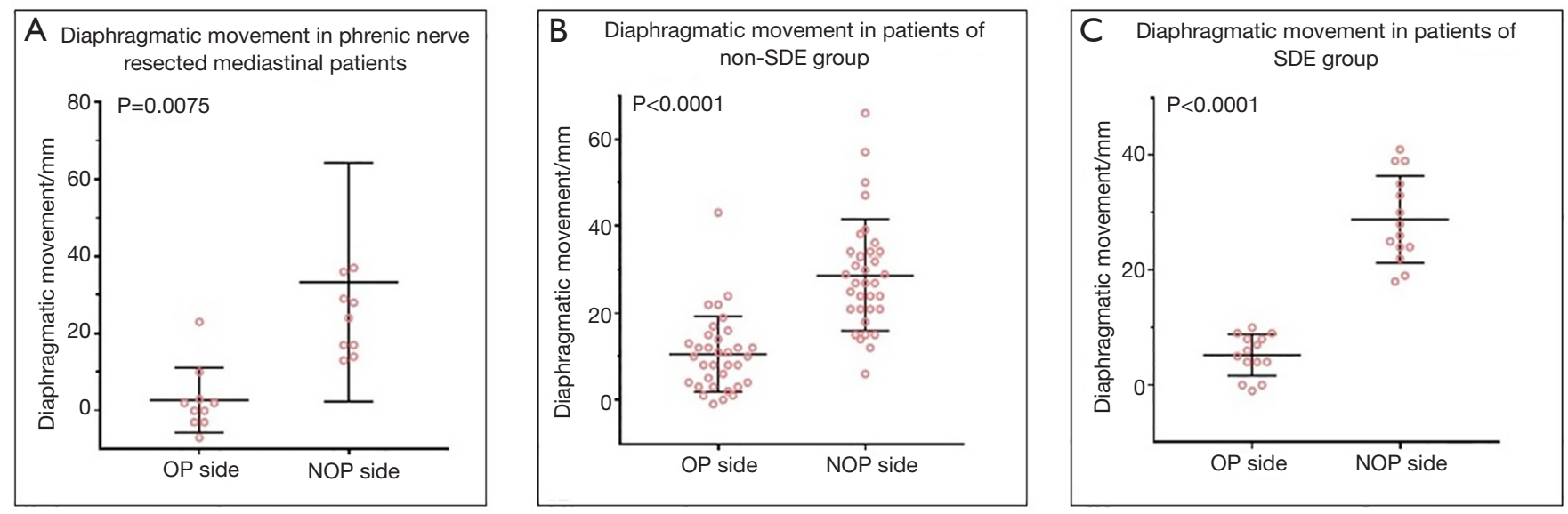

Figure 2 Extent of diaphragm movement on the operation and non-operation side on chest fluoroscopy after surgery. (A) Chest fluoroscopy in 10 thymoma-patients with phrenic nerve intentionally transected. Significant difference in diaphragmatic movement between OP and NOP sides was seen. (B,C) Chest fluoroscopy results in VATS lobectomy patients. In the non-SDE group, median diaphragm movement was $11.0 \mathrm{~mm}$ on the OP side and $28.7 \mathrm{~mm}$ on the NOP side, with a diaphragmatic movement ratio of $38 \%$ (B). In the SDE group however, median diaphragm movement was $5.0 \mathrm{~mm}$ on the OP side and $28.8 \mathrm{~mm}$ on the NOP side, with a diaphragmatic movement ratio of merely $17 \%(\mathrm{C})$.

lobectomy. Fluoroscopy test was carried out in 10 of these patients. Median diaphragm movement was $3.0 \mathrm{~mm}$ on the $\mathrm{OP}$ side and $33.3 \mathrm{~mm}$ on the NOP side $(\mathrm{P}=0.007$, Figure $2 A$ ), with a diaphragm movement ratio of $9 \%$.

\section{Incidence of SDE suggestive of PNI after VATS lobectomy}

According to the above results, SDE suggestive of inadvertent PNI was found in $56(7.4 \%)$ cases among 753 consecutive VATS lobectomy patients treated during the study period. As stated before, none of these patients had intentional phrenic nerve transection or noticed injury during surgery. The extent of diaphragm elevation was $15.8 \% \pm 1.3 \%$ in the non-SDE group and $35.9 \% \pm 1.5 \%$ in the SDE group, respectively $(\mathrm{P}=0.003)$. Fluoroscopy test was performed in 48 VATS lobectomy patients, 34 in the non-SDE group and 14 in the SDE group. In the non-SDE group, median diaphragm movement was $11.0 \mathrm{~mm}$ on the OP side and $28.7 \mathrm{~mm}$ on the NOP side (Figure $2 B$ ), with a diaphragmatic movement ratio of $38 \%$. In the SDE group however, median diaphragm movement was $5.0 \mathrm{~mm}$ on the OP side, and $28.8 \mathrm{~mm}$ on the NOP side (Figure $2 \mathrm{C}$ ), with a diaphragm movement ratio of merely $17 \%$. Significant difference in diaphragm movement was seen on the OP side between the SDE and the non-SDE groups $(\mathrm{P}=0.003$, Figure $3 A)$, but not on the NOP side $(\mathrm{P}=0.982$, Figure $3 B)$.
Significant difference was also found in diaphragmatic movement ratio between the SDE and the non-SDE groups $(\mathrm{P}$ value $=0.018$, Figure $3 C)$.

\section{Impact of SDE on postoperative course and post-operative pulmonary function change}

Patient demographics, preoperative co-morbidities, surgical procedures, and tumor characteristics were listed in Tables 1,2. Most of the patients were in good performance status before surgery. No significant difference was detected in gender, age, preoperative co-morbidity, or preoperative spirometry data between patients with or without SDE. No association was detected between SDE and side of OP or extent of lymph node harvesting. Neither was SDE found to be related to either $\mathrm{T}$ or $\mathrm{N}$ staging of the tumor. However, SDE was seen more often after upper lobectomies $(8.9 \%)$ than after middle or lower lobectomies (5.5\%), with a borderline significance $(\mathrm{P}=0.073)$. No patient died after surgery in this series. Incidence of postoperative morbidity was similar between patients with or without $\operatorname{SDE}(3.5 \%$ vs. $7.8 \%, \mathrm{P}=0.240)$. Similarly, length of hospital stay was not different between the two groups $(7.42 \pm 4.87 \mathrm{vs}$. $7.04 \pm 4.92$ days, $\mathrm{P}=0.574)$. Among 202 patients who had repeated spirometry test 6 months after surgery, significantly greater reduction in FEV1, FVC, and DLCO 

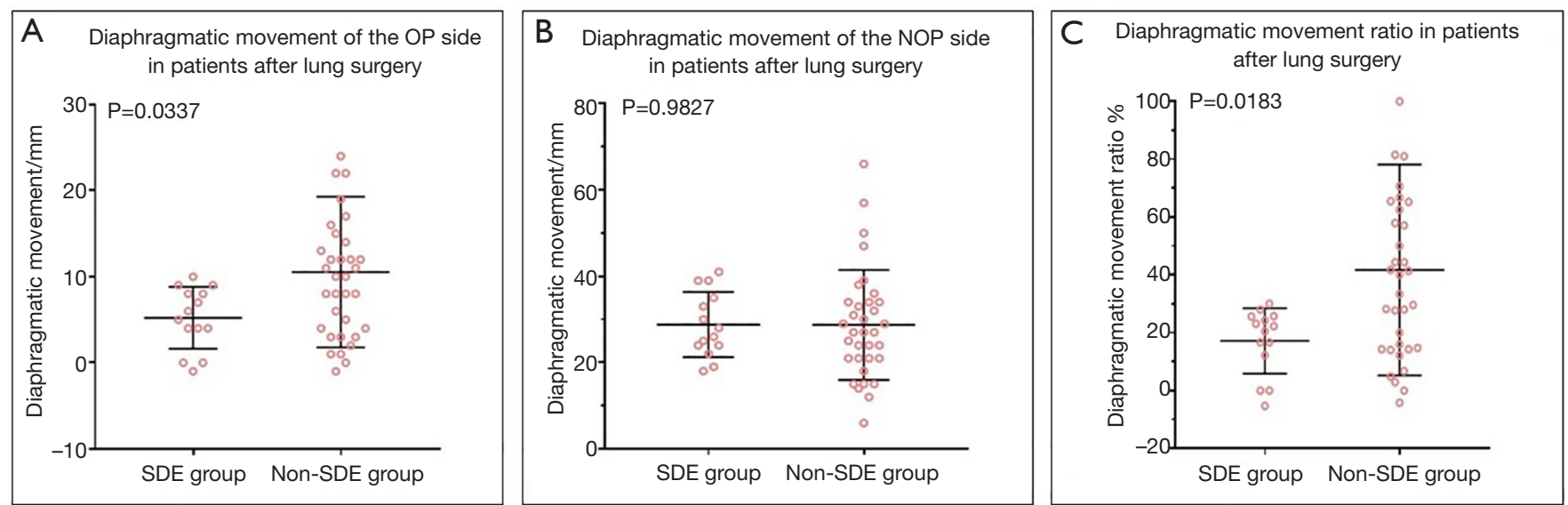

Figure 3 Comparison of diaphragm movement between the SDE and the non-SDE groups in VATS lobectomy patients. Significant difference in diaphragm movement was seen on the OP side between the SDE and the non-SDE group (A), but not on the NOP side (B). Furthermore, there was a significant difference in diaphragmatic movement ratio between the SDE and the non-SDE groups (C).

Table 1 Demographic characteristics of VATS lobectomy patients with and without significant diaphragm elevation

\begin{tabular}{|c|c|c|c|}
\hline Demographics & Patients with SDE, n (\%) & Patients without SDE, n (\%) & $P$ value \\
\hline Gender (male:female) & $25: 31$ & $322: 375$ & 0.822 \\
\hline Age at surgery (years) (mean $\pm S D)$ & $61.4 \pm 8.9$ & $59.6 \pm 9.2$ & 0.144 \\
\hline Comorbidity & & & 0.542 \\
\hline DM & $8(0.14)$ & $72(0.10)$ & \\
\hline COPD & $2(0.03)$ & $16(0.02)$ & \\
\hline $\mathrm{CHD}$ & $0(0)$ & $8(0.01)$ & \\
\hline Arrhythmia & $4(0.07)$ & $20(0.02)$ & \\
\hline
\end{tabular}

HBP, high blood pressure; DM, diabetes mellitus; COPD, chronic obstructive pulmonary disease; CHD, coronary heart disease; MT, malignant tumor; SDE, significant diaphragm elevation.

was detected in patients with SDE than those without (Table 3).

\section{Discussion}

Inadvertent PNI during lung cancer resection may be encountered when dissecting superior mediastinal lymph nodes or when mobilizing the hilum, close to where phrenic nerves are located. However, clinical diagnosis of PNI depends most often on subjective judgement. Comparing to preoperative chest $\mathrm{X}$-ray, obvious elevation of a hemi- diaphragm after surgery is often taken as a sign of PNI, with no specific criteria ever defined $(1,2)$. In fact, the individual variety of the patients' body size, the volume loss after lobectomy (with a compensatory diaphragm elevation to a certain extent) and the chest wall deformity after open thoracotomy is not carefully considered. To confirm the diagnosis of PNI, more specific diagnostic tests have been suggested $(1,6-11,17,18)$, including diaphragm ultrasound $(8,9,12)$, fluoroscopy $(1,7,11-13)$, magnetic stimulation of the phrenic nerves with evaluation of twitch trans-diaphragmatic pressure (10), respiratory function 
Table 2 Relationship between significant diaphragm elevation after VATS lobectomy and tumor location, $\mathrm{T}$ and $\mathrm{N}$ stage and extent of lymphadenectomy

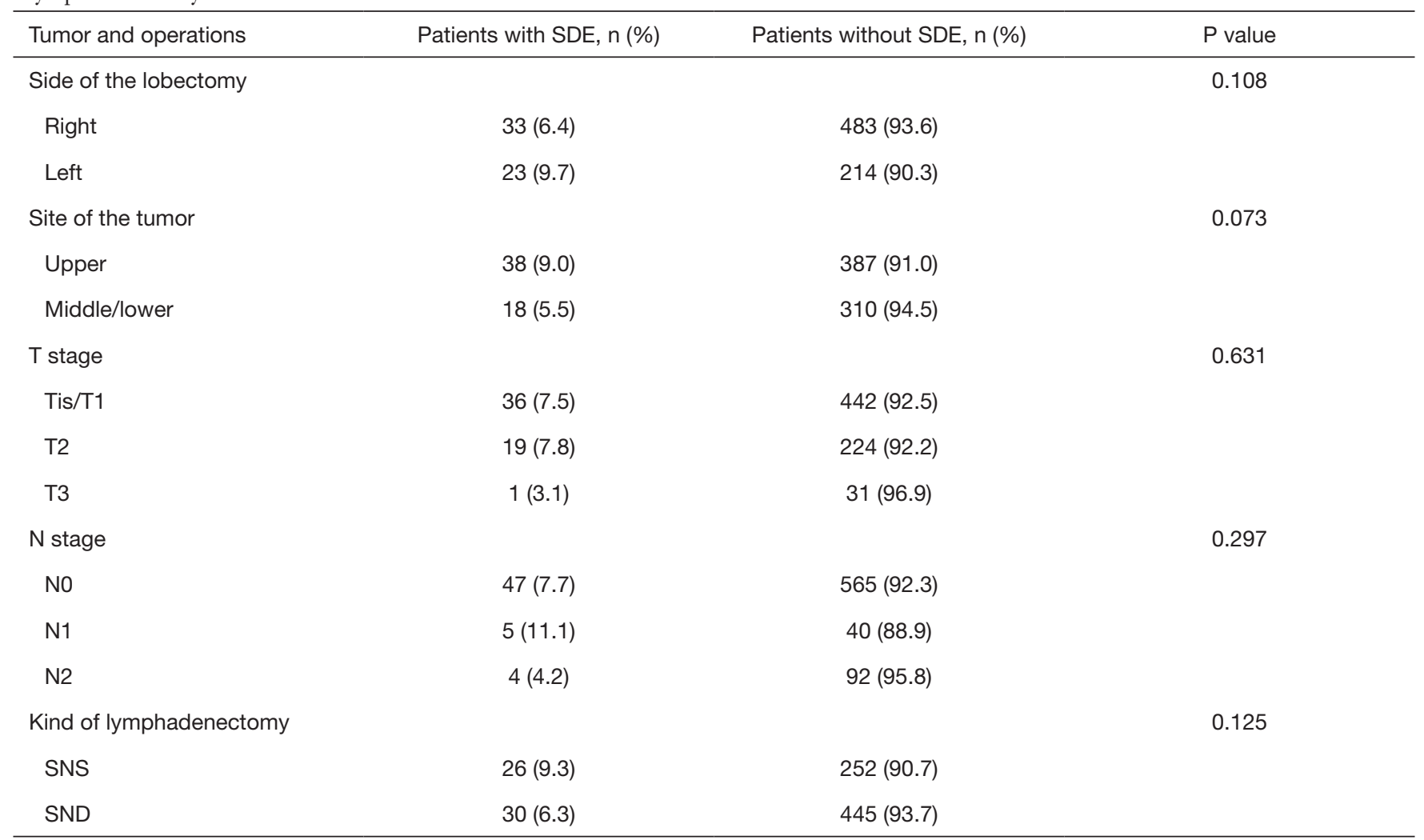

RUL, right upper lobe; RML, right middle lobe; RLL, right lower lobe; LUL, left upper lobe; LLL, left lower lobe; SNS, systemic lymph node sampling; SND, systemic lymph node dissection; SDE, significant diaphragm elevation.

Table 3 Rate of decline in FEV1, FVC and DLCO in VATS lobectomy patients with and without significant diaphragm elevation

\begin{tabular}{lccc}
\hline Spirometry parameters & Patients with SDE & Patients without SDE & P value \\
\hline Rate of decline in FEV1 (\%) & $36.1 \pm 19.5$ & $19.5 \pm 16.7$ & 0.000 \\
Rate of decline in FVC (\%) & $35.3 \pm 19.1$ & $17.4 \pm 14.6$ & 0.000 \\
Rate of decline in DLCO (\%) & $22.5 \pm 9.7$ & $14.9 \pm 12.1$ & 0.009 \\
\hline
\end{tabular}

FEV1, forced expiratory volume in 1 second; FVC, forced vital capacity; DLCO, diffusing capacity of the lung for carbon monoxide; SDE, significant diaphragm elevation.

tests (1), and electrodiagnostic studies like phrenic nerve conduction or diaphragm electromyography $(8,9,11,13,17)$. Unfortunately, these tests are not readily available in most of the thoracic surgery services.

In the current study, we first measured on chest X-ray the extent of diaphragm elevation in 22 patients receiving thymectomy for locally advanced thymic tumors. As phrenic nerve was intentionally divided because of tumor invasion in all these patients, hemidiaphragm paralysis was certain and would no doubt have caused SDE. In addition, there was also a lung volume loss to some extent because of concomitant big wedge resection. Considering that compensatory diaphragm elevation would be greater after a lobectomy than after a wedge resection, the $30 \%$ upper limit was taken as the criterion to define SDE suggestive of PNI after VATS lobectomy. Significant difference in diaphragmatic movement between the OP and the NOP sides was also verified by fluoroscopy test. Such measurement performed on chest X-ray offered us an objective and readily accessible approach to quantitatively 
evaluate the extent of diaphragm elevation and to help to diagnose inadvertent PNI in lung cancer surgery. Based on this criterion, we then investigated the incidence of SDE probably related to PNI in VATS lobectomy patients. Measurement of postoperative diaphragm elevation was carried out 6 months after surgery to rule out potential influences of pleural effusion, atelectasis, or pneumonia mostly seen in the early post-operative period (1) and to further improve the accuracy of diagnosing PNI. Fluoroscopy was again used as a validation test and diaphragm paralysis instead of simple elevation was verified (Figures 2,3), further confirming the reliability of this chest $\mathrm{X}$-ray diagnostic criterion.

As PNI after pulmonary resections has only been reported sporadically (6) and has been absent in most of previous studies on surgical outcomes, it is hard to decide whether this is unique to VATS procedures or it has a similar incidence in open surgery. Anyhow, our results indicate that SDE potentially due to inadvertent PNI is an underestimated condition after VATS lobectomy, with an incidence of $7.4 \%$ in our patients. As phrenic nerve was not transected in any of these patients, PNI was most likely caused by inadvertent injury when dissecting the anterior pulmonary hilum or upper mediastinal lymph nodes. The incidence was almost doubled after upper lobectomies $(8.9 \%)$ than after lower lobectomies $(5.5 \%)$ with a borderline statistical significance $(\mathrm{P}=0.073)$. The anatomy of the phrenic nerves may help to explain the difference, as they are close to the pulmonary hilum near the superior pulmonary veins. On the other hand, mediastinal lymph node dissection was not found to be associated with increased risk of SDE suggestive of PNI in this study, confirming what was reported in the ACOSOG Z0030 (19). Reasons for inadvertent PNI during pulmonary resections remain to be elucidated. Although some patient characteristics such as diabetes mellitus (18), chronic obstructive pulmonary diseases (COPD) (20), and increased age (21) have been considered as predisposing factors for PNI after cardiac procedures, no such correlation was found between these conditions and PNI in our patients. In open surgery, the authors use more "cold weapons" such as scissors and clamps, while in VATS pulmonary resections we rely mostly on energy devices such as hook cautery and harmonic scalpel (22). As lateral energy damage could be $7 \mathrm{~mm}$ from the currently available energy devices, care should be taken to stay away enough distance (at least 1 $\mathrm{cm}$ ) from the phrenic nerve to avoid potential thermal injury $(23,24)$.
In contrast to patient with bilateral diaphragm paralysis, unilateral PNI usually remains clinically asymptomatic in most of good risk surgical patients and only occasionally is associated with post-operative respiratory complications $(6,7)$. This may explain why not enough attention has been paid to PNI after lung cancer surgery, especially in the era of minimally invasive surgery, which reduces further the risk of postoperative functional complications. We also failed to find increased postoperative morbidities or prolonged hospital stay between patients with or without SDE, as most of our patients were good risk surgical candidates without severe comorbidity or seriously compromised cardiopulmonary function. However, we did notice that patients with SDE tended to complain dyspnea on exertion such as stair climbing or brisk walking, although we were not able to record detailed results due to the retrospective nature of this study. Moreover, a paralyzed diaphragm is bound to adversely affect the synchronicity and efficiency of its mechanical movement and thus to impair the respiratory function of the patient (25). By comparing spirometry data before and 6 months after surgery, we found a significantly greater reduction in postoperative FEV1, FVC, and DLCO in patients with SDE than those without. Our results are consistent with pulmonary function abnormalities in unilateral diaphragm paralysis described previously (25-33). And the extent of pulmonary function loss was much more significant comparing to our previous study results on spirometry changes in patients without major complications after VATS lobectomy (22). This further confirms that such SDE was due to inadvertent PNI rather than a mere compensatory change of lung volume loss after lobectomy.

Our study has certain limitations that need to be mentioned. As an uncontrolled retrospective study, it is bound to be affected by intrinsic selection biases. And although it would be ideal to make comparison between VATS and open surgery to see whether PNI was indeed caused by energy devices as we speculated, this is unfortunately not amenable as the vast majority of lung cancer surgery has been carried out under VATS at our hospital in the past decade. Also because of its retrospective nature, we were unable to check pulmonary function changes in every patient diagnosed of having SDE. In addition, we only studied diaphragm elevation on chest $\mathrm{X}$-ray and spirometry changes 6 months after surgery. It is likely that some additional patients might also have temporary PNI but recovered within the 6 months period. Although this may have caused an underestimation of the 
true incidence of PNI, it would have a little clinical impact, as we did not find increased morbidity or respiratory problems in the early postoperative period. Moreover, once the diaphragm function is recovered, it would no longer have a significant negative impact on patients' quality of life. Certainly, it would be interesting to continue following up those patients with SDE suggestive of PNI to see whether there would be a chance to recover later. Further studies are needed to evaluate the exact impact of PNI on patients' quality of life and oncologic outcomes.

In conclusion, our results suggest that inadvertent PNI during VATS lobectomy is an underestimated phenomenon and is associated with significantly greater loss of pulmonary function. Patients with more than $30 \%$ diaphragm elevation on chest films after VATS lobectomy are highly likely to have PNI and should undergo fluoroscopic validation. Enough attention should be paid to avoid inadvertent PNI so that lung cancer patients may benefit from minimally invasive surgery to a greater extent.

\section{Acknowledgments}

Funding: None.

\section{Footnote}

Reporting Checklist: The authors have completed the STROBE reporting checklist. Available at http://dx.doi. org/10.21037/tlcr-20-540

Data Sharing Statement: Available at http://dx.doi. org/10.21037/tlcr-20-540

Conflicts of Interest: All authors have completed the ICMJE uniform disclosure form (available at http://dx.doi. org/10.21037/tlcr-20-540). The authors have no conflicts of interest to declare.

Ethical Statement: The authors are accountable for all aspects of the work in ensuring that questions related to the accuracy or integrity of any part of the work are appropriately investigated and resolved. All procedures performed in this study were in accordance with the Declaration of Helsinki (as revised in 2013). The study design was approved by the Local Ethics Committees. All patients gave a written informed consent for the surgical treatment and were aware that all information could be used anonymously for scientific purpose only.
Open Access Statement: This is an Open Access article distributed in accordance with the Creative Commons Attribution-NonCommercial-NoDerivs 4.0 International License (CC BY-NC-ND 4.0), which permits the noncommercial replication and distribution of the article with the strict proviso that no changes or edits are made and the original work is properly cited (including links to both the formal publication through the relevant DOI and the license). See: https://creativecommons.org/licenses/by-nc-nd/4.0/.

\section{References}

1. Deng Y, Byth K, Paterson HS. Phrenic nerve injury associated with high free right internal mammary artery harvesting. Ann Thorac Surg 2003;76:459-63.

2. Tripp HF, Sees DW, Lisagor PG, et al. Is phrenic nerve dysfunction after cardiac surgery related to internal mammary harvesting? J Card Surg 2001;16:228-31.

3. Wheeler WE, Rubis LJ, Jones CW, et al. Etiology and Prevention of Topical Cardiac Hypothermia induced Phrenic Nerve Injury and Left Lower Lobe Atelectasis during Cardiac Surgery. Chest 1985;88:680-3.

4. Esposito RA, Spencer FC. The effect of pericardial insulation on hypothermic phrenic nerve injury during open-heart surgery. Ann Thorac Surg 1987;43:303-8.

5. Kohno T. Management of complications in thoracoscopic surgery. J Thorac Dis 2018;10:S1620-23.

6. Ostrowska M, De Carvalho M. Prognosis of phrenic nerve injury following thoracic interventions: four new cases and a review. Clin Neurol Neurosurg 2012;114:199-204.

7. Laub GW, Muralidharan S, Chen C, et al. Phrenic nerve injury. A prospective study. Chest 1991;100:376-9.

8. Merino-Ramirez MA, Juan G, Ramón M, et al. Electrophysiologic evaluation of phrenic nerve and diaphragm function after coronary bypass surgery: prospective study of diabetes and other risk factors. J Thorac Cardiovasc Surg 2006;132:530-6.

9. DeVita MA, Robinson LR, Rehder J, et al. Incidence and natural history of phrenic neuropathy occurring during open heart surgery. Chest 1993;103:850-6.

10. Swallow EB, Dayer MJ, Oldfield WL, et al. Right hemidiaphragm paralysis following cardiac radiofrequency ablation. Respir Med 2006;100:1657-9.

11. Wilcox PG, Paré PD, Pardy RL. Recovery after unilateral phrenic injury associated with coronary artery revascularization. Chest 1990;98:661-6. 
12. Olopade CO, Staats BA. Time course of recovery from frostbitten phrenic after coronary artery bypass graft surgery. Chest 1991;99:1112-5.

13. Cruz-Martinez A, Armijo A, Fermoso A, et al. Phrenic nerve conduction study in demyelinating neuropathies and open-heart surgery. Clin Neurophysiol 2000;111:821-5.

14. Bendixen M, Jørgensen OD, Kronborg C, et al. Postoperative pain and quality of life after lobectomy via video-assisted thoracoscopic surgery or anterolateral thoracotomy for early stage lung cancer: a randomised controlled trial. Lancet Oncol 2016;17:836-44.

15. Mei J, Guo C, Xia L, et al. Long-term survival outcomes of video-assisted thoracic surgery lobectomy for stage I-II non-small cell lung cancer are more favorable than thoracotomy: a propensity scorematched analysis from a high-volume center in China. Transl Lung Cancer Res. 2019;8:155-66.

16. Miller MR, Hankinson J, Brusasco V, et al. Standardisation of spirometry. Eur Respir J 2005;26:319-38.

17. Dimopoulou I, Daganou M, Dafni U, et al. Phrenic nerve dysfunction after cardiac operations: electrophysiologic evaluation of risk factors. Chest 1998;113:8-14.

18. Kohorst WR, Schonfeld SA, Altman M. Bilateral diaphragmatic paralysis following topical cardiac hypothermia. Chest 1984;85:65-8.

19. Allen MS, Darling GE, Pechet TT, et al. Morbidity and mortality of major pulmonary resections in patients with early-stage lung cancer: initial results of the randomized, prospective ACOSOG Z0030 trial. Ann Thorac Surg 2006;81:1013-9; discussion 1019-20.

20. Cohen A, Katz M, Katz R, et al. Chronic obstructive pulmonary disease in patients undergoing coronary artery bypass grafting. J Thorac Cardiovasc Surg 1995;109:574-81.

21. Norris AHN, Shock W, Wagman IH. Age changes in the maximal conduction velocity of motor fibers of human ulnar nerves. J Appl Physiol 1953;5:589-93.

22. Gu Z, Wang $\mathrm{H}$, Mao T, et al. Pulmonary function changes after different extent of pulmonary resection under video-assisted thoracic surgery. J Thorac Dis 2018;10:2331-7.
23. Chen C, Cavanaugh JM, Kallakuri S, et al. Acute Effects of Ultrasonic Shears and Monopolar Electrosurgery on Sciatic Nerve Electrophysiology. $\mathrm{Br}$ J Med Med Res 2016;14:1-8.

24. Phillips CK, Hruby GW, Durak E, et al. Tissue response to surgical energy devices. Urology 2008;71:744-8.

25. Clague HW, Hall DR. Effect of posture on lung volume: airway closure and gas exchange in hemidiaphragmatic paralysis. Thorax 1979;34:523-6.

26. Ross Russell RI, Helms PJ, Elliott MJ. A prospective study of phrenic nerve damage after cardiac surgery in children. Intensive Care Med 2008;34:728-34.

27. Agostini E, Sant'Ambrogio G. The diaphragm. In: Campbell E, Agostini E, Newson DJ. editors. The respiratory muscles, mechanics and neurological control. London, England: Lloyd-Luke, 1970:145-60.

28. Ugalde P, MiroS, Provencher S, et al. Ipsilateral diaphragmatic motion and lung function in long term pneumonectomy patients. Ann Thorac Surg 2008;86:1745-51.

29. Easton PA, Fleetham JA, de la Rocha A, et al. Respiratory function after paralysis of the right hemidiaphragm. Am Rev Resp Dis 1983;127:125-8.

30. Ridyard JB, Stewart RM. Regional lung function in unilateral diaphragmatic paralysis. Thorax 1976;31:438-42.

31. Graham DR, Kaplan D, Evans CC, et al. Diaphragmatic plication for unilateral diaphragmatic paralysis: a 10-year experience. Ann Thorac Surg 1990;49:248-51.

32. Wright CD, Williams JG, Ogilvie CM, et al. Results of diaphragmatic plication for unilateral diaphragmatic paralysis. J Thorac Cardiovasc Surg 1985;90:195-8.

33. Bugge AS, Lund MB, Valberg M, et al. Cause-specific death after surgical resection for early-stage nonsmall-cell lung cancer. Eur J Cardiothorac Surg 2018;53:221-7.

Cite this article as: Ventura L, Zhao W, Chen T, Wang Z, Feng J, Gu Z, Ji C, Fang W. Significant diaphragm elevation suggestive of phrenic nerve injury after thoracoscopic lobectomy for lung cancer: an underestimated problem. Transl Lung Cancer Res 2020;9(5):1822-1830. doi: 10.21037/tlcr-20-540 